\title{
Influência das Correntes de Soldagem do Processo Plasma-MIG sobre a Geometria do Cordão de Solda e Taxa de Fusão do Arame
}

\author{
(Influence of Welding Current in Plasma-MIG Weld Process on the Bead Weld Geometry and Wire Fusion Rate)
}

\author{
André Alves de Resende ${ }^{1}$, Valtair Antonio Ferraresi ${ }^{1}$, Américo Scotti ${ }^{1}$, Jair Carlos Dutra ${ }^{2}$ \\ ${ }^{1}$ Universidade Federal de Uberlândia, Faculdade de Engenharia Mecânica, Centro para Pesquisa e Desenvolvimento de \\ Processos de Soldagem, Campus Santa Mônica, 38400 902, Uberlândia, MG, aaresende@gmail.com,valtairf@mecanica.ufu.br, \\ ascotti@mecanica.ufu \\ ${ }^{2}$ Universidade Federal de Santa Catarina, Faculdade de Engenharia Mecânica, Labsolda, Campus Trindade, 88040-970, \\ Florianópolis, SC,jdutra@labsolda.ufsc.br
}

\begin{abstract}
Resumo
Uma das versões do processo Plasma-MIG consiste basicamente da combinação de um arco Plasma com um arco MIG/MAG em uma única tocha. Com essa associação, se procura unir vantagens individuais de cada arco. A principal característica consiste na independência entre o aporte de energia imposto pelo processo e o material adicionado, resultando em uma maior facilidade em atuar sobre a geometria do cordão de solda. Na literatura corrente existem poucas informações relacionadas com o processo, além de que a maioria remota principalmente das décadas de 70 e 80, quando a tecnologia disponível não era capaz de viabilizar o processo para a indústria da época. No entanto, nos últimos anos, a difusão das novas fontes eletrônicas utilizadas em soldagem contribuiu na retomada do interesse pelo processo Plasma-MIG. Neste contexto, este trabalho objetivou ampliar os estudos relacionados à influência do balanço das correntes Plasma e MIG/MAG sobre a geometria do cordão de solda e taxa de fusão do arame. Soldagens de simples deposição sobre chapa forma realizadas com uma combinação de corrente de Plasma e de MIG/MAG em 3 níveis cada, mantendo-se, pela correção da velocidade de soldagem, o mesmo volume de cordão. Foi observado que a introdução da corrente Plasma sobre a corrente MIG/MAG reduz a penetração e a diluição e proporciona cordões menos convexos. Por outro lado, o uso da corrente Plasma faz aumentar a taxa de fusão do arame MIG/MAG. Entretanto, parece que a intensidade da corrente Plasma não é o fator governante nestas alterações.
\end{abstract}

Palavras-chave: Soldagem;Plasma-MIG;Arco Hibrido.

\begin{abstract}
One of the versions of the Plasma-MIG process is basically a combination of a Plasma arc with a MIG/MAG arc in a single torch. With this association, the advantages of each arc are searched. The main characteristic of it is the independence between the heat input by the process and the deposited material, resulting in greater facility for control the bead weld geometry. In current literature, there are shortage of information related to the process, and most of them remote from 70's and 80's years, when the available technology was not able to make the process viable for the industry. However, in the last years, the diffusion of new electronic power sources used in welding brought up again the interest in Plasma-MIG process. In this context, this work aimed to contribute to the studies related to the influence of the MIG and Plasma current balance on the geometry of the bead weld and wire fusion rate. Bead-on-plates weld were carried out with a Plasma and MIG/MAG current combinations at 3 levels each, keeping, by welding speed corrections, the bead volume the same. It was observed that the introduction of the Plasma current over the MIG/MG current reduces penetration and dilution and lead to less convex beads. On the other hand, the use of Plasma current increases the MIG/MAG wire fusion rate. However, it seems that the intensity of the Plasma current is not the governing parameter on those changes.
\end{abstract}

Key-words: Welding; Plasma-MIG; Hybrid Arc.

\section{Introdução}

Nos últimos anos, com os avanços de novas tecnologias disponíveis para o desenvolvimento de processos de soldagem e a procura por processos com maior capacidade de produção e produtividade, surgiram os chamados "Processos Híbridos de Soldagem", com destaque ao MIG-Laser e Plasma-MIG. Trata-se da associação física de dois processos, com o intuito de

(Recebido em 29/07/2009; Texto Final em 25/11/2009).

Publicado com permissão da ABCM - Associação Brasileira de Engenharia e Ciências Mecânicas - Artigo originalmente apresentado no $5^{\circ}$ COBEF, Belo Horizonte, MG, Abril de 2009 
aproveitar as características mais atrativas de cada [1].

Como atrativo, o processo $\mathrm{MIG} / \mathrm{MAG}$ oferece uma alta taxa de deposição, a possibilidade de adição de material com diferentes composições químicas. Entretanto, no processo MIG/MAG a penetração é relativamente pequena, exigindo normalmente a confecção de chanfros. Esta desvantagem é superada pela adição de material. Porém, como nos outros processos com eletrodos consumíveis, a corrente de soldagem está estreitamente interligada com a taxa de alimentação de metal de adição, para uma dada condição de soldagem (gás de proteção, comprimento e tipo de eletrodo, etc.). Este fato traduz a forte dependência existente entre os aportes de energia e de material nesses processos, ou seja, há pouca margem para se aumentar a corrente sem também alterar a quantidade de material a ser depositado. Como a geometria de um cordão de solda depende da energia imposta, e também da quantidade de material depositado por unidade de comprimento da solda, uma das principais conseqüências desta dependência é a dificuldade em se controlar livremente a geometria do cordão de solda. $\mathrm{O}$ Plasma, por sua vez, é um processo com arco bastante estável, e no modo com alimentação de arame, não há estrita dependência entre seu arco e o material alimentado.

Uma das versões do processo de Soldagem Plasma-MIG pode ser definida como a combinação dos arcos Plasma do processo MIG/MAG em uma única tocha, onde o metal de adição é alimentado pelo orifício do bocal plasma. O processo pode ser usado tanto para soldagem quanto para revestimento [2]. Ele une as vantagens de alta produtividade dos processos de soldagem a arco aos menores custos dos equipamentos, proporcionados pelas possibilidades de combinação entre as fontes de corrente comercialmente disponíveis para a soldagem a Plasma e MIG/ MAG [3].

A Figura 1 mostra de forma esquemática uma tocha de soldagem do processo Plasma-MIG, destacando os principais componentes. Uma particularidade pode ser observada no eletrodo Plasma, que para o Processo Plasma-MIG é de cobre e possui um formato anular, diferente daquele utilizado no processo Plasma convencional, que é constituído de Tungstênio e possui um formato pontiagudo. Com a utilização de eletrodos de cobre (emissão por campo e não termiônica), a parte Plasma pode operar em polaridade positiva, com tendência a promover maior estabilidade na porção MIG/MAG do processo. Com esta configuração, também é possível a utilização de misturas de gases ativos, uma vez que o cobre é menos suscetível a degradação pelo oxigênio presente nas misturas ativas. Outro ponto a ser considerado é que o eletrodo de cobre possui uma área maior para dissipar o calor e também conta com um sistema de refrigeração auxiliar. Naturalmente, o bocal constritor de Plasma passa a ter um orifício maior que do processo convencional, para permitir a passagem, também de forma concêntrica, do arame, o que resulta num arco menos concentrado.

Nos processos de soldagem a arco elétrico, a abertura do arco pode ser conseguida de forma artificial, basicamente de três maneiras: alto diferencial de tensão, alta freqüência-alta tensão ou curto circuito. Um alto diferencial de tensão em baixa freqüência é inviável, pois oferece riscos aos operadores. Pulsos de alta tensão (ou alta tensão em alta frequiência) são extremamente danosos para equipamentos eletrônicos (emissão de ruídos). Finalmente, a abertura por curto circuito gera uma apreciável quantidade de respingos, que podem alcançar partes vitais da tocha, danificando a mesma. Tendo em vista estas dificuldades, no inicio da década de 80 foi proposto por Essers et al. [5] um novo método de acendimento do arco para o processo Plasma-MIG, chamado "Soft Start". Nesta forma de acendimento do arco, o arame-eletrodo é utilizado para gerar um arco de baixa intensidade, através do qual ocorre a formação do arco plasma [4]. Com este método, a abertura do arco ocorre praticamente isenta de respingos, evitando com que partes vitais da tocha sejam danificadas.

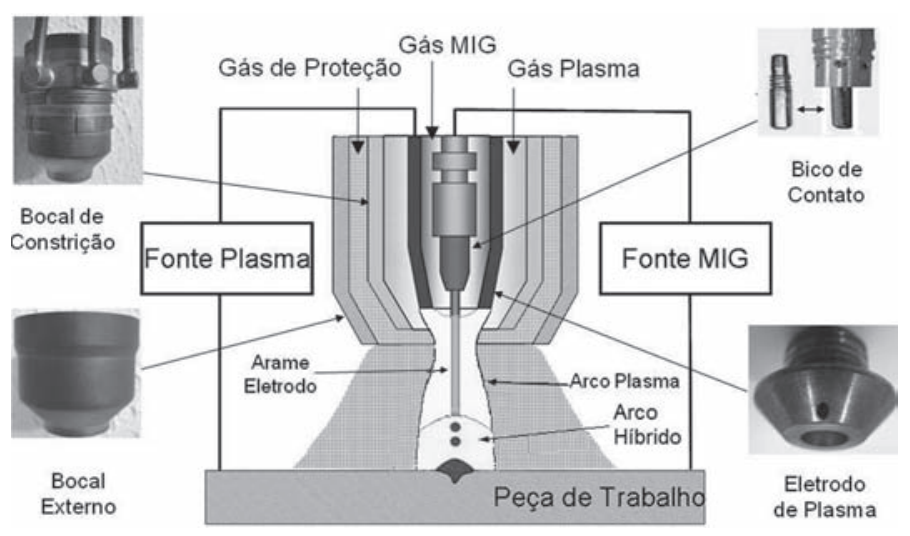

Figura 1. Desenho esquemático de uma tocha para soldagem Plasma-MIG, com destaque para os componentes principais (adaptado de [4]).

A Figura 2 ilustra as seis etapas básicas para acendimento do arco pelo método "Soft Start", a saber:

Etapa 1: As duas fontes apresentam tensão em vazio e o arame-eletrodo da porção MIG/MAG do processo é alimentado em direção à peça.

Etapa 2: Ao toque do arame na peça, o movimento do mesmo é interrompido e a fonte gera um arco de baixa intensidade de corrente (em torno de $35 \mathrm{~A}$ ), sem fusão considerável do arame.

Etapa 3: O alimentador do arame inverte a rotação e o arame retrocede em direção à tocha de soldagem, trazendo progressivamente o arco para dentro da tocha, atingido as proximidades do eletrodo do Plasma.

Etapa 4: Como a fonte Plasma já possui tensão em vazio, ocorre o acendimento imediato do arco-plasma, devido à atmosfera ionizada pelo arco MIG/MAG de baixa potência.

Etapa 5: Após o acendimento do arco Plasma, o arco MIG/ MAG é extinto (corte de energia) com o objetivo de impedir a transferência metálica e de proporcionar um pré-aquecimento no início da junta somente com a energia do arco-plasma.

Etapa 6: O arame volta a se deslocar em direção à peça de trabalho e, como a fonte MIG/MAG apresenta novamente tensão em vazio e o meio está ionizado pelo arco Plasma, ocorre o reacendimento espontâneo do arco MIG/MAG, sem necessidade de curto-circuito (isso garante um início de cordão de solda livre de respingos), mas já com a corrente regulada para a operação de soldagem. 
Para entender os mecanismos com que a energia térmica é transferida à peça e os mecanismos que governam a penetração no processo Plasma-MIG, Essers e Walter [6] utilizaram a técnica de calorimetria e observaram que existem diferenças entre as energias absorvidas pela peça para os processos MIG/ MAG e Plasma-MIG. As diferenças foram explicadas pelo fato da existência de dois ânodos no processo Plasma-MIG, sendo um a extremidade do eletrodo do MIG/MAG e outra do eletrodo Plasma, que neste caso, parte do calor gerado no eletrodo Plasma é absorvida pelo sistema de refrigeração (fato que leva a variação no perfil do cordão de solda quando comparados). Jelmorini et al. [7] avaliaram o perfil do cordão de solda do processo PlasmaMIG e mostraram que a presença da corrente Plasma modifica o perfil do mesmo no sentido de aumentar a largura e promover uma melhor molhabilidade.

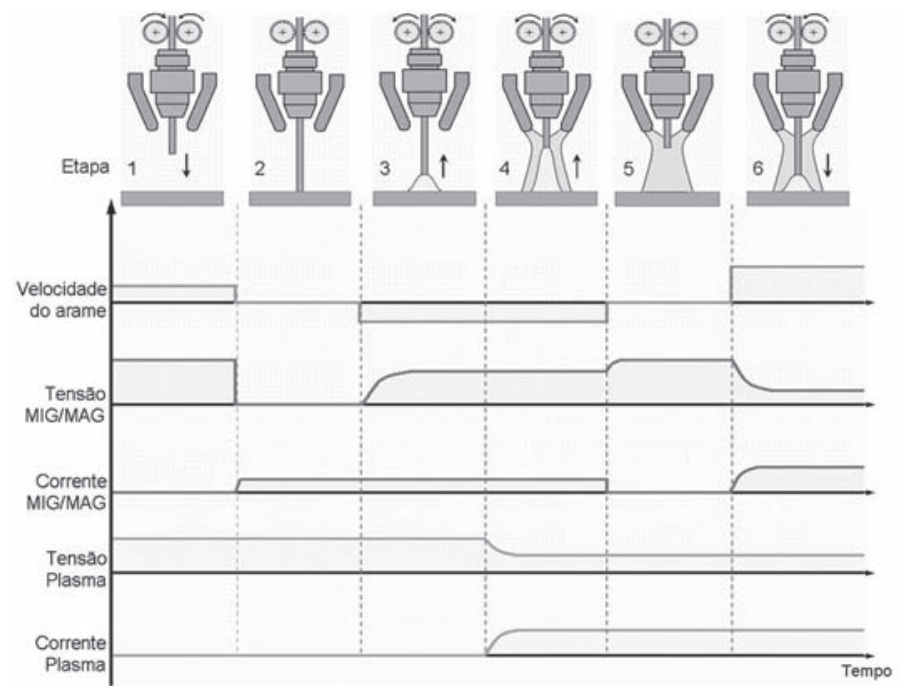

Figura 2. Esquema de funcionamento do "Soft Start", (Adaptado de [1] e [4]).

Pelo exposto, parece ser consenso que a associação entre as correntes do Plasma e do MIG/MAG pode oferecer a possibilidade de atuar sobre a geometria do cordão de solda ao variar o balanço entre as correntes. Porém, as explicações acima citadas da literatura sobre a influência das correntes dos circuitos Plasma e MIG/MAG sobre a geometria do cordão ainda são vagas e pouco quantificadas, além do que, remontam principalmente das décadas de 70 e 80 , quando a tecnologia disponível não era capaz de viabilizar o processo para a indústria da época. Além disto, a concepção da tocha utilizada naquela época era diferente, com o eletrodo Plasma pontiagudo, resultando num arco constrito, diferente do eletrodo anular utilizado atualmente, que produz um arco menos concentrado. No entanto, Oliveira [4] aponta que nos últimos anos a difusão das novas fontes eletrônicas utilizadas em soldagem, que permitem um melhor controle na fusão e transferência de material, através da utilização de diferentes formas de onda de corrente, contribuiu de forma decisiva na retomada do interesse pelo processo Plasma-MIG, devido a sua grande potencialidade. Desta forma, faz-se necessário o desenvolvimento de novas tecnologias e conhecimentos acerca do processo. Neste contexto, este trabalho objetiva ampliar os estudos relacionados à influência dos balanços do circuito Plasma e do MIG/MAG sobre a geometria do cordão de solda e taxa de fusão do arame.

\section{Procedimento Experimental}

Um total de nove experimentos foram realizados para avaliar a influência das correntes do processo Plasma-MIG na geometria do cordão de solda. Na realização de tais experimentos, foram utilizadas uma tocha comercial para soldagem Plasma-MIG refrigerada a água e duas fontes eletrônicas multiprocesso do tipo chaveada no secundário. Uma das fontes foi programada para trabalhar no modo MIG/MAG e outra no modo Plasma, ambas com característica estática em corrente constante, para se garantir os valores de corrente em todos os experimentos comparativos. Neste modo, regulam-se os valores das correntes e da velocidade de alimentação, sendo as tensões resultantes da carga (caracterizada por cada arco). Para a operação de abertura do arco, foi utilizado o procedimento 'Soft Start' (utilizado para iniciar o arco hibrido do processo Plasma-MIG).

Como o valor da velocidade de alimentação varia com a corrente Plasma e, principalmente, com a corrente MIG/MAG, a velocidade de soldagem também foi ajustada para cada experimento. A velocidade de alimentação foi obtida de forma a se obter um comprimento de arco aproximadamente constante para a mesma relação Velocidade de Soldagem/Velocidade de Alimentação. Esta regulagem foi realizada aumentando-se gradativamente a velocidade de alimentação até que o arame tocasse na poça (gerando curtos-circuitos). A partir desse ponto, a velocidade de alimentação era então reduzida suavemente até que os curtos-circuitos deixassem de existir, tendo uma transferência goticular com um comprimento de arco pequeno e, aproximadamente, constante em todos os ensaios.

A bancada experimental está apresentada na Figura 3. A tocha Plasma-MIG é acoplada às fontes que normalmente são utilizadas para os processos que o originaram (Plasma e MIG/ MAG), necessitando apenas de um comando único para as duas fontes. Uma interface microprocessada faz a seqüência de abertura do arco (Soft Start) e comanda os parâmetros préregulados para o processo. Um item de grande importância e que merece destaque, é o sistema de refrigeração (temperatura controlada de água, por exemplo, abaixo da temperatura ambiente), indicado na figura como item 4 . A temperatura da água de refrigeração foi regulada para ficar em torno de $17^{\circ} \mathrm{C}$. $\mathrm{O}$ uso apenas dos sistemas convencionais de resfriamento (água circulante por radiadores) se mostraram não serem eficientes para evitar danos à tocha, com destaque para o bocal constritor e eletrodo anular de cobre.

As distâncias de posicionamento dos principais elementos da tocha estão apresentadas no esquema da Figura 4, onde DTP é a distância da tocha a peça, RP o recuo do eletrodo Plasma e RM o recuo do bico de contato MIG/MAG. Os valores de RP e RM são fixos e dependem da característica de construção da tocha que correspondem, respectivamente, a 9 e $18 \mathrm{~mm}$. Já o valor de DTP pode ser variado e, para este trabalho, foi utilizado $9 \mathrm{~mm}$, resultando em uma distância do bico de contato MIG/MAG à peça (DBCP) de $27 \mathrm{~mm}$. Se forem utilizados valores de DTP 
muito pequenos (para permitir menores DBCP), o destacamento da gota de metal fundido pode acontecer no interior da tocha, o que pode causar problemas de desvio destas gotas para a tocha.

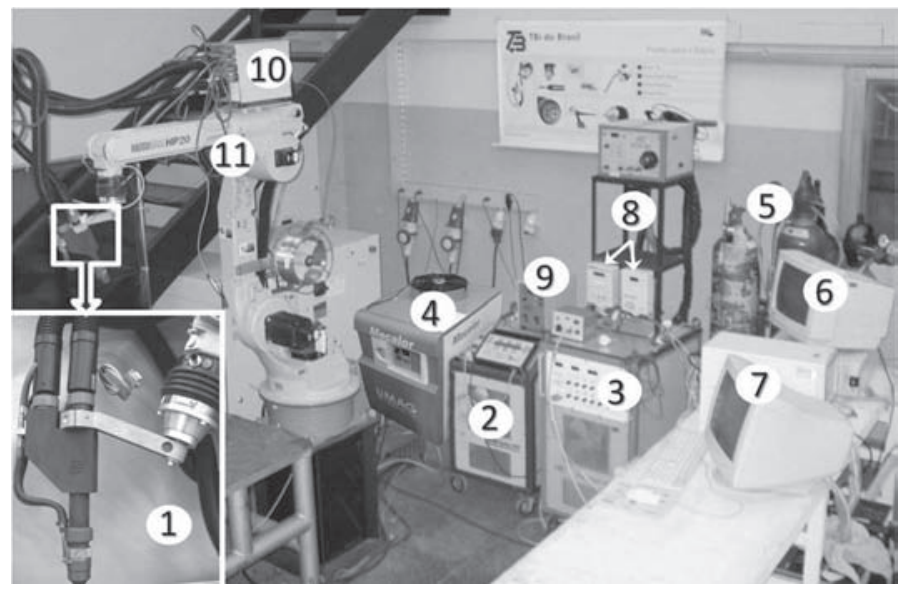

Figura 3. Bancada experimental utilizada, onde: 1 - Tocha Plasma-MIG; 2 - Fonte MIG/MAG; 3 - Fonte Plasma; 4 Unidade de refrigeração de água; 5 - Gases para o processo; 6 - Sistema de controle para o processo; 7 - Sistema de aquisição de sinais elétricos; 8 - Medidores de vazão para os gases Plasma e MIG/MAG; 9 - Interface responsável pelo controle do cabeçote alimentador; 10 - Cabeçote alimentador; 11 - Robô.

Para efeito de comparação, todos os ensaios foram realizados como simples deposição sobre chapa ("bead-onplate"). As chapas de teste foram confeccionadas a partir de chapas de aço carbono ABNT 1020 nas dimensões 300 x 50,8 x $6,35 \mathrm{~mm}$. O arame-eletrodo utilizado nos ensaios foi da classe AWS ER70S-6, de diâmetro 1,2 mm. Ar+4\% CO como gás MIG/MAG a 5 1/min, Ar como gás Plasma a 5 1/min e Ar como gás de Proteção a 15 1/min. A escolha dos gases e vazão foi feita de forma subjetiva, com indicação de adequabilidade em testes exploratórios (não houve intenção de otimização e nem de estuda da influência deste parâmetro). Optou-se por trabalhar com valores de corrente MIG/MAG acima da corrente de transição globular-goticular típicas para o processo MIG/MAG convencional, em três níveis (200, 240 e 280 A). Para a corrente Plasma, também foram avaliados três níveis (0, 60 e 100 A). Os ensaios atenderam a uma combinação das correntes, como mostra a Tabela 1. A velocidade de soldagem foi regulada para manter a mesma relação velocidade de soldagem pela velocidade de alimentação, com o intuito de se obter uma mesma quantidade de material depositado por unidade de comprimento e comparar o efeito das variáveis sobre os perfis dos cordões de solda.

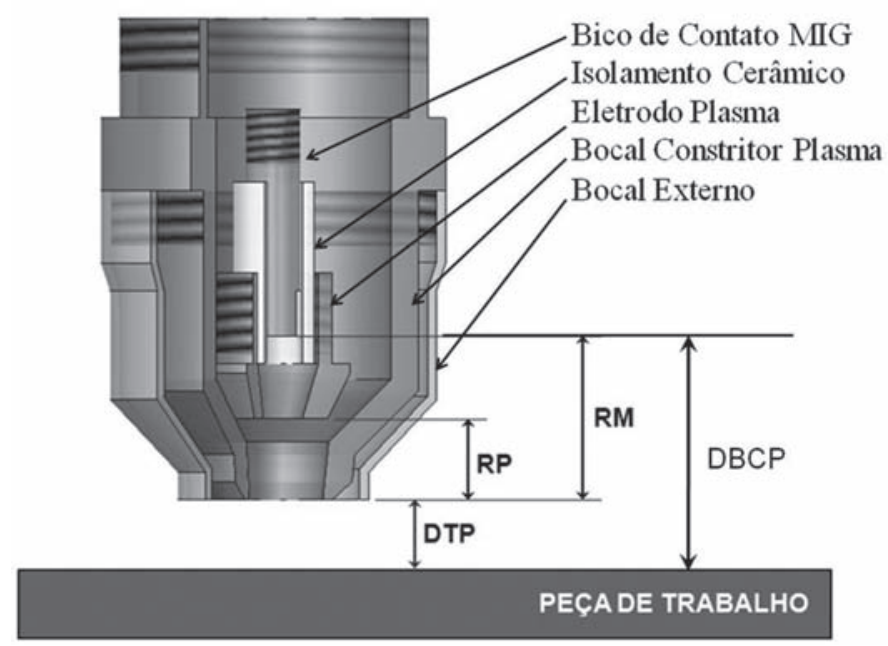

Figura 4. Vista esquemática do posicionamento dos principais elementos da tocha Plasma-MIG. Onde DTP é a distância da tocha a peça, RP o recuo do eletrodo Plasma e RM o recuo do bico de contato MIG/MAG.

Tabela 1. Parâmetros de regulagem para os Testes.

\begin{tabular}{|c|c|c|c|c|c|}
\hline Teste & $\begin{array}{c}I_{M I G / M A G} \\
{[\mathrm{~A}]}\end{array}$ & $\begin{array}{c}I_{\text {PLASMA }} \\
{[\mathrm{A}]}\end{array}$ & Vsold $[\mathrm{m} / \mathrm{min}]$ & Valim $[\mathrm{m} / \mathrm{min}]$ & $\left(\right.$ Vsold/Valim) $* 10^{2}$ \\
\hline 1 & 200 & 0 & 0,30 & 5,0 & 6,0 \\
\hline 2 & 200 & 60 & 0,42 & 7,1 & 5,9 \\
\hline 3 & 200 & 100 & 0,45 & 7,4 & 6,1 \\
\hline 4 & 240 & 0 & 0,44 & 7,3 & 6,0 \\
\hline 5 & 240 & 60 & 0,65 & 10,8 & 6,0 \\
\hline 6 & 240 & 100 & 0,67 & 11,2 & 5,9 \\
\hline 7 & 280 & 0 & 0,53 & 8,8 & 6,0 \\
\hline 8 & 280 & 60 & 0,77 & 12,7 & 6,0 \\
\hline 9 & 280 & 100 & 0,80 & 13,0 & 6,1 \\
\hline
\end{tabular}


Os ensaios 1, 4 e 7 foram realizados sem corrente passando pelo circuito Plasma, ou seja, se trata de soldagem MIG/MAG com corrente constante utilizando a tocha Plasma-MIG. Estes ensaios são os pontos de referência para verificar a influência da corrente plasma no processo e, principalmente, na geometria do cordão. Estes ensaios em particular, foram realizados com a fonte Plasma desligada, conseqüentemente o procedimento 'Soft Start' não foi utilizado e os parâmetros MIG/MAG foram regulados diretamente no painel de controle da Fonte. Tal procedimento foi executado para garantir que a corrente MIG/ MAG circulasse somente através do arco MIG/MAG, uma vez que se o procedimento 'Soft Start' fosse utilizado com a corrente Plasma regulada para $0 \mathrm{~A}$ (zero A), a fonte Plasma permaneceria com tensão em vazio durante a soldagem e uma pequena parcela da corrente MIG/MAG circularia através do circuito Plasma. Os demais testes foram realizados com corrente passando pelo circuito Plasma e com a utilização do 'Soft Start'.

Para aquisição de corrente, utilizou-se, como transdutor/ condicionador de sinal, um sensor Hall e para aquisição da tensão de soldagem, um divisor de tensão. Ambos fornecem como saída um sinal de tensão na faixa de $\pm 10 \mathrm{~V}$, que corresponde exatamente à faixa de medição da placa de aquisição de resolução de 14 bits utilizada. Para uma faixa de medição do sensor Hall de $\pm 500 \mathrm{~A}$, a resolução de medição resultante, calculada pela razão (faixa de medição do sensor hall/resolução da placa) foi de 0,06 A para a corrente. De maneira similar, a faixa de medição de $\pm 100 \mathrm{~V}$ do divisor de tensão resultou numa resolução de medição de 0,01 $\mathrm{V}$ para a tensão.

O efeito sobre a geometria foi estudado por meio de técnicas convencionais de macrografia, sobre seções transversais tiradas das placas de teste soldadas (cordão de $250 \mathrm{~mm}$ ). Para cada placa de teste, foram cortadas duas seções transversais, uma aproximadamente no meio e uma próxima ao final do cordão (20 $\mathrm{mm}$ da extremidade). As medidas dos parâmetros geométricos foram realizadas através de imagens digitais com resolução de 20 pixels por milímetros, o que resulta numa resolução de medição de $0,05 \mathrm{~mm}$ para os parâmetros geométricos.

\section{Resultados e Discussão}

Na Tabela 2 têm-se os valores de tensão e corrente monitorados durante os testes para os circuitos Plasma e MIG/ MAG, com seus respectivos desvios padrões. Na Tabela 3 se apresenta as seções transversais representativas de cada um das chapas de teste soldadas. Para cada chapa de teste, foram retiradas duas seções transversais, uma aproximadamente no meio e uma próxima ao final do cordão. Os valores médios com os respectivos desvios padrões das respectivas geometrias dos cordões estão apresentados na Tabela 4.

Tabela 2. Valores médios dos sinais de corrente e tensão monitorados para os circuitos Plasma e MIG/MAG.

\begin{tabular}{ccccc}
\hline Teste & $\begin{array}{c}I_{\text {MIG } / M A G} \\
{[\mathrm{U}]}\end{array}$ & $\begin{array}{c}U_{\text {MIG } / M A G} \\
{[\mathrm{~V}]}\end{array}$ & $\begin{array}{c}I_{\text {PLASMA }} \\
{[\mathrm{A}]}\end{array}$ & $\begin{array}{c}U_{\text {PLASMA }} \\
{[\mathrm{V}]}\end{array}$ \\
\hline 1 & $200,7^{ \pm 4,0}$ & $30,4^{ \pm 2,0}$ & $*$ & $*$ \\
2 & $202,1^{ \pm 3,8}$ & $27,6^{ \pm 3,1}$ & $55,1^{ \pm 7,6}$ & $37,5^{ \pm 3,6}$ \\
3 & $201,4^{ \pm 3,9}$ & $29,6^{ \pm 2,9}$ & $93,2^{ \pm 7,7}$ & $33,1^{ \pm 4,7}$ \\
4 & $240,4^{ \pm 3,9}$ & $26,7^{ \pm 1,9}$ & $*$ & $*$ \\
5 & $241,6^{ \pm 3,7}$ & $27,7^{ \pm 4,8}$ & $54,8^{ \pm 5,1}$ & $36,4^{ \pm 6,7}$ \\
6 & $242,1^{ \pm 3,8}$ & $22,5^{ \pm 3,5}$ & $94,2^{ \pm 7,5}$ & $30,8^{ \pm 2,1}$ \\
7 & $279,3^{ \pm 3,9}$ & $28,9^{ \pm 1,5}$ & $*$ & $*$ \\
8 & $281,5^{ \pm 3,8}$ & $25,0^{ \pm 2,4}$ & $55,3^{ \pm 4,8}$ & $33,4^{ \pm 2,0}$ \\
9 & $281,1^{ \pm 3,8}$ & $24,1^{ \pm 2,0}$ & $94,2^{ \pm 4,9}$ & $30,8^{ \pm 2,0}$ \\
\hline
\end{tabular}

Obs: Valores mascados com asterisco $(*)$ correspondem às soldagens com a fonte Plasma desligada. 
Tabela 3. Seções transversais da placas de teste soldadas (para efeito de escala, a espessura da chapa está visível e corresponde à 6,35 $\mathrm{mm})$.

\begin{tabular}{llll}
\hline & $I_{M I G / M A G}=200 \mathrm{~A}$ & $I_{M I G / M A G}=240 \mathrm{~A}$ & $I_{M I G / M A G}=280 \mathrm{~A}$ \\
\hline$I_{\text {PLASMA }}=0 \mathrm{~A}$ & \\
$I_{\text {PLASMA }}=60 \mathrm{~A}$ &
\end{tabular}

Tabela 4. Parâmetros geométricos medidos para os cordões de solda.

\begin{tabular}{ccccc}
\hline Teste & Largura $[\mathrm{mm}]$ & Reforço $[\mathrm{mm}]$ & Penetração $[\mathrm{mm}]$ & Área Fundida $\left[\mathrm{mm}^{2}\right]$ \\
\hline 1 & $9,25^{ \pm 0,10}$ & $2,83^{ \pm 0,04}$ & $1,66^{ \pm 0,13}$ & $8,66^{ \pm 0,23}$ \\
2 & $9,62^{ \pm 0,16}$ & $2,50^{ \pm 0,14}$ & $1,41^{ \pm 0,13}$ & $9,05^{ \pm 0,61}$ \\
3 & $9,78^{ \pm 0,11}$ & $2,30^{ \pm 0,17}$ & $1,14^{ \pm 0,06}$ & $8,06^{ \pm 0,39}$ \\
4 & $8,35^{ \pm 0,35}$ & $2,92^{ \pm 0,26}$ & $2,60^{ \pm 0,13}$ & $11,85^{ \pm 0,49}$ \\
5 & $9,28^{ \pm 0,11}$ & $2,43^{ \pm 0,17}$ & $1,73^{ \pm 0,11}$ & $8,04^{ \pm 0,65}$ \\
6 & $9,39^{ \pm 0,31}$ & $2,59^{ \pm 0,11}$ & $1,48^{ \pm 0,24}$ & $7,02^{ \pm 0,37}$ \\
7 & $8,39^{ \pm 0,12}$ & $3,29^{ \pm 0,15}$ & $3,00^{ \pm 0,28}$ & $12,19^{ \pm 2,41}$ \\
8 & $9,19^{ \pm 0,08}$ & $2,57^{ \pm 0,10}$ & $2,23^{ \pm 0,04}$ & $8,81^{ \pm 0,62}$ \\
9 & $9,47^{ \pm 0,16}$ & $2,62^{ \pm 0,26}$ & $2,18^{ \pm 0,12}$ & $9,20^{ \pm 1,48}$ \\
\hline
\end{tabular}

Obs: A área fundida e penetração se referem às medidas tomadas na região abaixo da superfície da placa de teste.

A Figura 5 apresenta a variação da penetração, área fundida e diluição com as correntes de soldagem. Deve-se lembrar de que o aumento da corrente foi acompanhado por um aumento proporcional da velocidade de soldagem. Pela Figura 5(a), é observado um aumento na penetração com o aumento da corrente MIG/MAG, justificável pelo aumento da pressão que o arco exerce sobre a poça fundida devido à maior concentração dos campos magnéticos. Outro fato, observado por Scotti \& Rodrigues [8], é que maiores correntes resultam em gotas com maiores velocidades, o que pode contribuir, pelo momentum, para uma maior penetração.

Ainda na Figura 5(a), percebe-se uma tendência clara da redução da penetração do cordão de solda ao se inserir corrente no circuito Plasma. Já pela Figuras 5(b) e 5(c), percebe-se uma tendência clara da redução da área fundida (abaixo da linha que delimita a superfície da chapa, não incluindo a área relativa ao material depositado) e da diluição (fator correlacionados) do cordão de solda ao se inserir corrente no circuito Plasma. Como apresentado anteriormente, o arco híbrido possui uma maior área de contato com a peça, conseqüentemente maior a área aquecida, maior a molhabilidade e maior é a largura do cordão. Como a imposição de corrente no circuito Plasma aumenta consideravelmente o volume do arco híbrido em relação ao arco MIG/MAG (Plasma $=0 \mathrm{~A}$ ), a pressão causada pelos campos magnéticos pode se reduzir, já que a corrente está menos concentrada, resultando, dessa forma, em gotas com menores velocidades. Um indicativo para essa redução na concentração da corrente é citado no trabalho de Ton [9], que, por meio de 


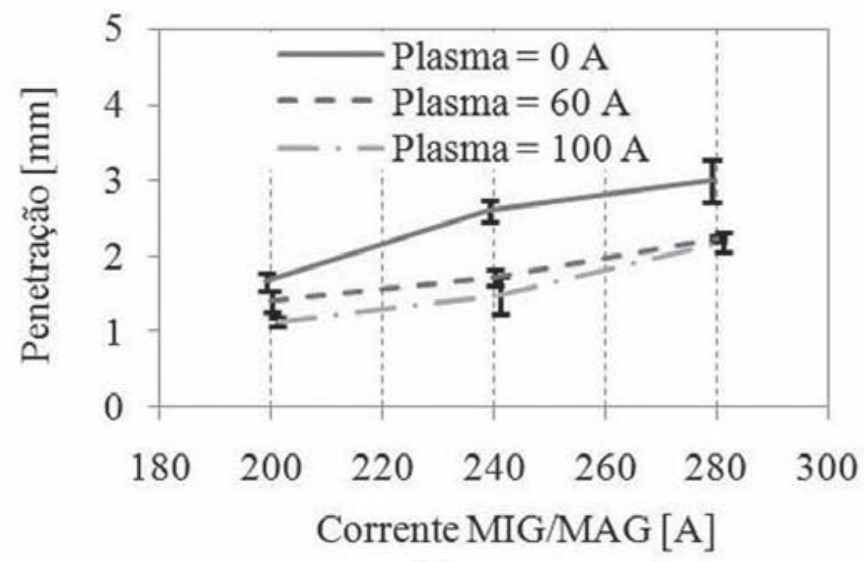

(a)

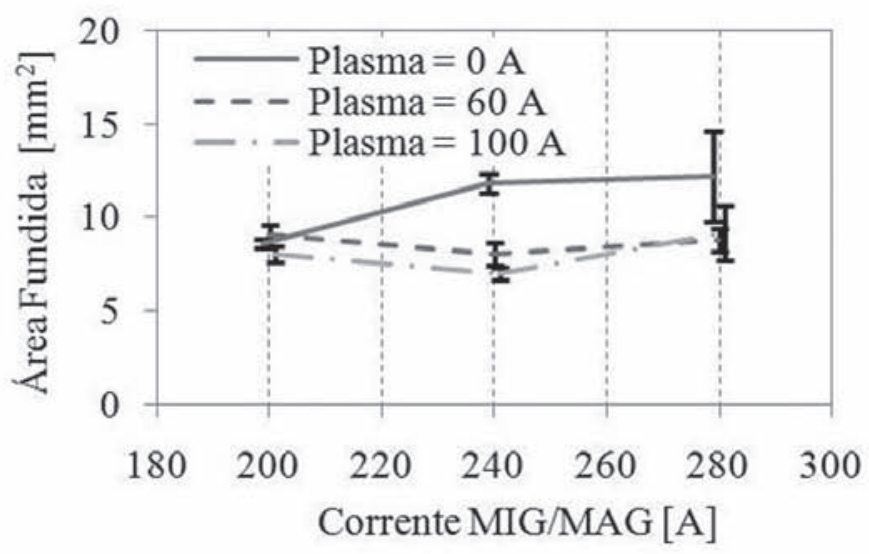

(b)

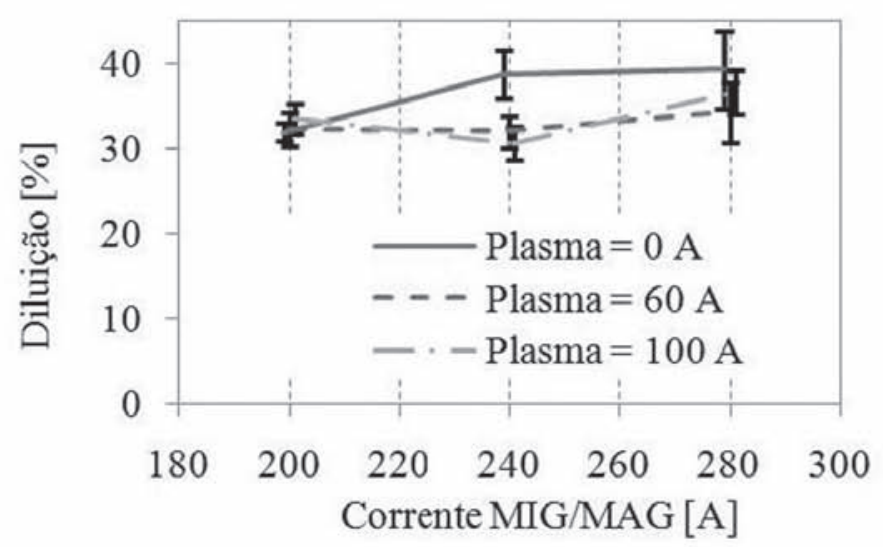

(c)

Figura 5. Penetração (a), Área Fundida (b) e Diluição (c) do cordão de solda em função da corrente MIG/MAG para três níveis de corrente plasma.

técnicas de espectroscopia óptica, verificou que a maior parte da corrente que passa pelo arame (corrente MIG/MAG) flui através da periferia do arco híbrido. Essers e Walter [6] observaram, através de técnicas de fotografia a alta velocidade, que para valores altos de corrente (acima de $170 \mathrm{~A}$ ) no arame há a tendência de rotacionar a ponta do eletrodo, mas não chegando a caracterizar uma transferência do tipo rotacional. Isto faz com que as gotas sejam transferidas para a poça de fusão, mas de maneira menos concentrada, o que, por consequiência, pode reduzir a penetração e aumenta a largura do cordão. Assim, uma explicação para a diminuição na penetração seria pela redução do momentum (massa x velocidade) das gotas impingindo sobre a poça. Percebe-se também pela figura mencionada (Figura 5(a)) que maiores diferenças de penetração acontecem para maiores correntes, confirmado pelo comportamento da área fundida (Figura 5(b)) e da diluição (Figura 5(c))

A Figura 6 apresenta a variação da largura e do reforço com as correntes de soldagem. Percebe-se uma tendência de aumentar a largura do cordão de solda e de reduzir o reforço ao se inserir corrente no circuito Plasma. Esse efeito pode ser atribuído ao aumento da área de contato do arco híbrido com a peça em comparação ao arco MIG/MAG, que, por conseqüência, aumenta a área da superfície da chapa aquecida, aumentando a molhabilidade da mesma, resultando em cordões mais largos e mais rasos (lembrar que o volume era o mesmo). Por outro lado, um aumento na corrente Plasma de 60 A para 100 A não provocou alteração significativa na largura e reforço. Provavelmente o efeito foi pequeno, porque o volume de gás ionizado, correspondente ao arco hibrido, já é relativamente grande e um incremento de $40 \mathrm{~A}$ de corrente Plasma não é capaz de proporcionar um aumento significativo no volume do arco hibrido, conseqüentemente sobre a área de contato do mesmo com a peça. Sendo assim, a maior parte do calor que é inserido com o incremento da corrente Plasma é perdido para o ambiente, reduzindo a contribuição desse aumento de corrente na fusão da chapa.

A Figura 7 apresenta a relação entre velocidade de alimentação e corrente de soldagem. Observa-se uma contribuição significativa do arco Plasma na fusão do eletrodo; para uma corrente Plasma de $60 \mathrm{~A}$, ocorreu um ganho de quase $3 \mathrm{~m} / \mathrm{min}$ na velocidade de alimentação do arame. Este resultado foi similar ao obtido por Oliveira [4] soldando com Plasma-MIG pulsado. Por outro lado, o aumento da corrente Plasma de 60 A para 100 A não provocou um incremento significativo na velocidade de alimentação. Essa tendência também coincide com a observada por Oliveira [4], que afirma que o incremento na taxa de fusão 


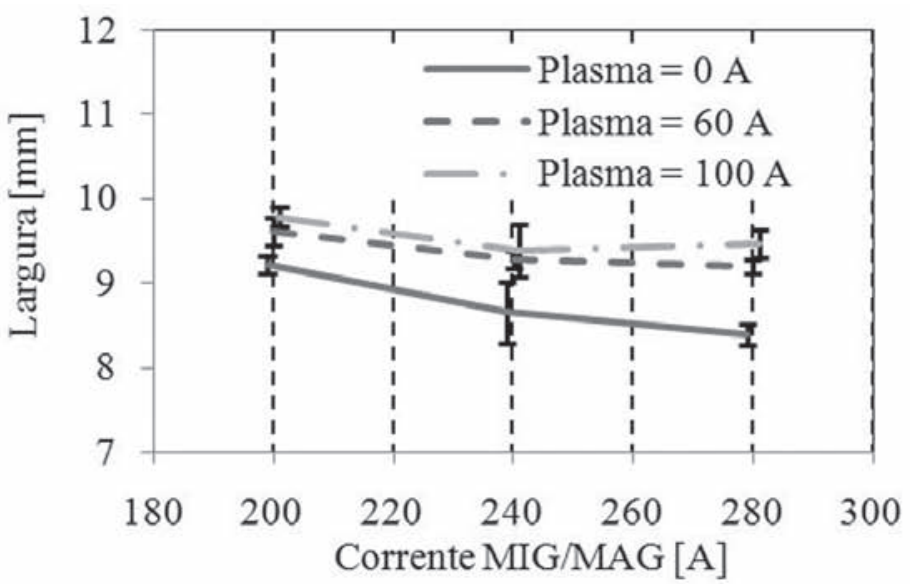

(a)

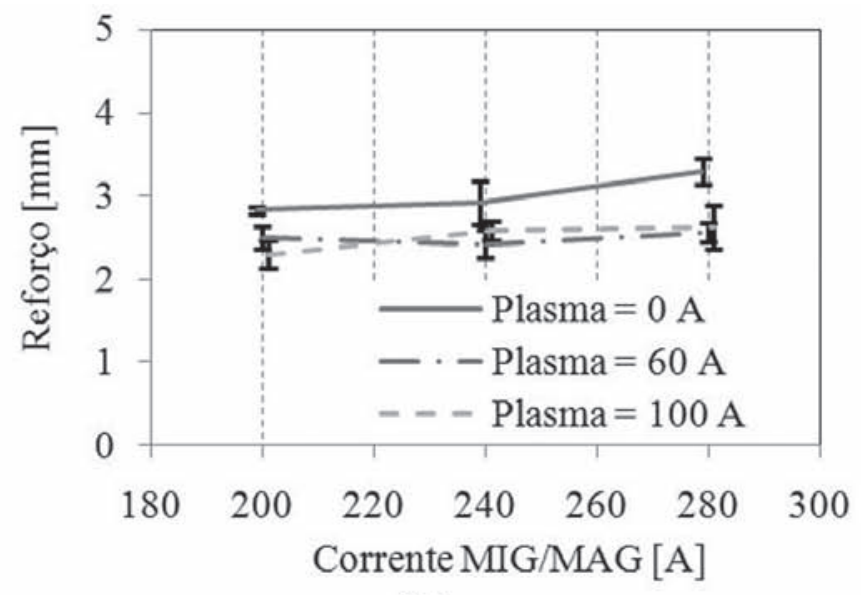

(b)

Figura 6. Largura (a) e Reforço (b) do Cordão de Solda em função da corrente MIG/MAG para três níveis de corrente Plasma.

do arame causada pelo arco Plasma depende da capacidade do arame eletrodo absorver a energia disponibilizada pelo arco Plasma e que essa absorção ocorre pelos três mecanismos de transferência de calor: radiação, convecção e condução.

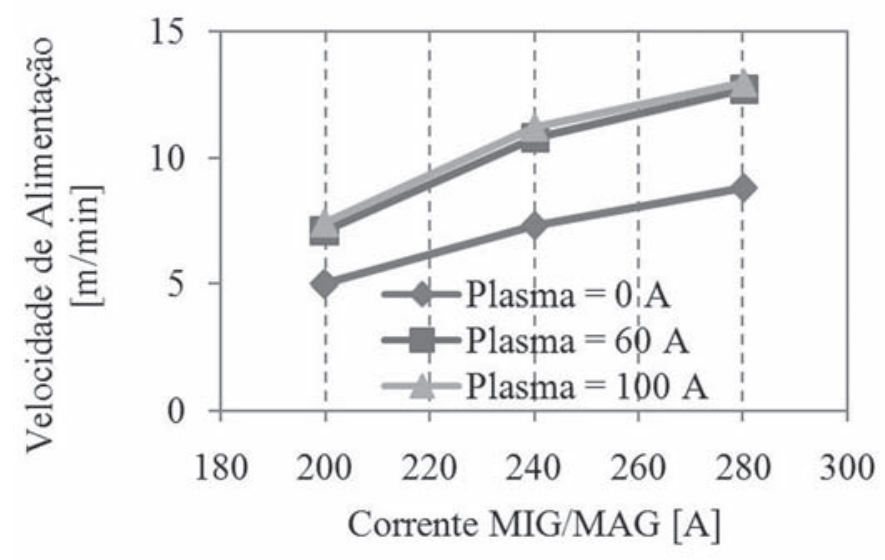

Figura 7. Velocidade de alimentação de arame em função da corrente MIG/MAG para três níveis de Corrente Plasma.

\section{Conclusões}

Para as condições e parâmetros de soldagem utilizados neste trabalho, pode-se concluir que:

- Diferentes combinações entre as correntes do Plasma e do MIG/MAG produzem cordões com diferentes dimensões, que seguem a tendência de diminuição da penetração, da área fundida e da diluição (contrariando a expectativa dos usuários, devido a presença do termo Plasma no nome do processo) e do aumento da largura e redução do reforço quando se introduz a corrente Plasma, indicando que o processo possui potencialidades para ser aplicado em operações que exijam uma menor diluição, como por exemplo, em revestimentos e soldagem de chapas finas;

- O uso da corrente Plasma faz ainda aumentar a taxa de fusão do arame para uma mesma corrente MIG/MAG, indicando a possibilidade de aumento da capacidade de produção do processo.
- O valor da corrente Plasma em si, para os valores estudados, não parece ser o fator governante destas mudanças, e sim o uso da corrente Plasma.

\section{Agradecimentos}

Os autores agradecem Faculdade de Engenharia Mecânica da UFU, através de seu Centro para Pesquisa e Desenvolvimento de Processos de Soldagem (Laprosolda), pela oportunidade de utilização dos equipamentos e do suporte técnico disponibilizado, e a agências FAPEMIG (proc. TEC 1763/06), pela concessão de recursos financeiros, e CNPq, pela concessão de bolsas de estudo e pesquisa para os autores.

\section{Referências}

[1] REIS, R.; SCOTTI, A. Fundamentos e prática da soldagem a plasma. Artliber Editora, $1^{a}$ ed., 2007. ISBN 978-85-8809839-8. 147p.

[2] HARRIS, I. D. Plasma MIG Welding. In: ASM Metals HandBook Volume 6 - Welding, Brazing, and Soldering, pp. 605-615. 1994.

[3] MATTHES, K.J; KOHLER, T. Electrical effects and influencing quantities in the case of the hybrid plasma-MIG welding process. Welding and Cutting. p. 87 - 90. Feb. 2006. [4] OLIVEIRA, M. A. Desenvolvimento do processo de soldagem híbrido Plasma-MIG para operações de soldagem e brasagem. Tese de Doutorado, Universidade Federal de Santa Catarina, Florianopolis, SC. 2006. 148f.

[5] ESSERS, W.G. et al. Plasma-MIG welding - a new torch and arc starting method. Metal Construction. p 36-42. Jan. 1981.

[6] ESSERS, W. G.; WALTER, R. Heat Transfer and Penetration Mechanisms with GMA and Plasma-GMA Welding. Welding Journal, vol. 60, pp. 37-42, 1981.

[7] JELMORINI, G; TICHELAAR, G. W; ESSERS, W. G. Welding characteristics of the Plasma-MIG process. Metal Construction, nov. 1975. p. $568-572$.

[8] SCOTTI, A. \& RODRIGUES, C.E.A.L., Determination of momentum as a mean of quantifying the mechanical energy 
delivered by droplets during MIG/MAG welding, Eur. Phys.

J. Appl. Phys. 45 (1), 2009, pp 11201, p1-p8, DOI: 10.1051/

epjap:2008196

[9] TON, H. Physical properties of the Plasma-MIG welding arc.

Applied Physics, vol 8 (4). 1975. p. 922-933. 